Cómo citar este trabajo: Durán Vian, F., Serrano-Martínez, M., \& Pons Izquierdo, J. J. (2018). Recuperación del paisaje fluvial y su revalorización social: el caso de Arévalo (Ávila). Boletín de la Asociación de Geógrafos Españoles, 78, 419-443. doi: http://dx.doi.org/10.21138/bage.2719

\title{
Recuperación del paisaje fluvial y su revalorización social: el caso de Arévalo (Ávila)
}

Recovery and social revaluation of the fluvial landscape: the case of Arévalo (Ávila)

\author{
Francisco Durán Vian \\ fduranvian@gmail.com \\ Miriam Serrano-Martínez \\ miriserrano@unav.es \\ Juan José Pons Izquierdo \\ jpons@unav.es \\ Área de Geografía y Ordenación del Territorio \\ Universidad de Navarra (España)
}

\section{Resumen}

El presente artículo muestra la reconciliación entre los ciudadanos de Arévalo (Ávila, España) y sus ríos, a través de la ejecución de un proyecto de mejora y acondicionamiento paisajístico. Las claves del éxito de la recuperación de este espacio fluvial, antes degradado y abocado al olvido, fueron tres, fundamentalmente. En primer lugar, se confeccionó un meditado proyecto de recuperación paisajística de escasa dotación presupuestaria, cuya ejecución se realizó satisfactoriamente. En segundo lugar, la idea de actuar en el paisaje fluvial de este pueblo recibió un importante impulso político. Por último, cabe destacar la sensibilidad ambiental con la que se desarrolló el proyecto en todas sus fases, así como la involucración de la propia ciudadanía, a quien se hizo partícipe en todo momento del proceso de ejecución. La transformación del paisaje y su revalorización, así 
como la recuperación del patrimonio natural y cultural ligado a los ríos de Arévalo, han mejorado la calidad de vida de sus habitantes. La metodología empleada, que es replicable para futuras intervenciones en esta o en otras localidades, así como los resultados más relevantes, se exponen en el presente artículo.

Palabras clave: rehabilitación paisajística; recuperación fluvial; parque fluvial; participación ciudadana; educación ambiental.

\begin{abstract}
This article shows the reconciliation between the Arévalo (Avila, Spain) citizens and their rivers, achieved through the execution of a project of improvement and reconditioning of the landscape. The keys of success for the recovery of this fluvial space, previously degraded and forgotten, were three. Firstly, a planned project with low budget allocation was satisfactorily carried out. In second place, the idea of acting in the town's fluvial landscape received an important political stimulus. Finally, it is important to highlight the environmental sensitivity with which the project was developed through all its phases, as well as the involvement of the citizens at all times. Furthermore, the transformation of the landscape and its enhancement, as well as the recovery of the natural and cultural heritage linked to the rivers, have improved the quality of life of its inhabitants. The methodology used, which is replicable for future interventions in this and other localities, as well as the most relevant results, are presented in this article.
\end{abstract}

Key words: landscape rehabilitation; fluvial restoration; fluvial park; citizen participation; environmental education.

\title{
1 La relación del ser humano con el paisaje fluvial
}

Los ríos son uno de los principales factores que favorecieron el asentamiento territorial de los pueblos y ciudades. Por este motivo, muchos cursos fluviales atraviesan el corazón de las ciudades y, hoy en día, se han convertido en la verdadera columna vertebral de su sistema de áreas verdes. Estos espacios ejercen, en el ámbito urbano y periurbano, un importante papel de regulación microclimática y del ciclo del agua, favorecen la biodiversidad, actúan como depuradores de la contaminación atmosférica y atenuantes del ruido (Comisión Europea, 2014). Asimismo, cumplen también funciones sociales, recreativas, de ocio, deportivas, educativas, terapéuticas, estéticas, turísticas e incluso laborales (Bolund \& Hunhammar, 1999; Comisión Europea, 2011). Por todo ello, contribuyen a potenciar la calidad de vida urbana (Macdonald, 2007).

A pesar de que estos servicios ecosistémicos son bien conocidos en la actualidad, muchas ciudades aún conciben sus ríos como elementos aislados y separados de su realidad. Son numerosos los casos en los que el paisaje fluvial ha perdido su identidad como consecuencia del 
desarrollo urbanístico y de la falta de sensibilización y respeto hacia él (Cuello, 2009). Además, su planificación y gestión -sobre todo en el ámbito periurbano- no siempre es adecuada, dando lugar a espacios poco apacibles, deshabitados y, en definitiva, marginados (Fernández \& Yáñez, 2015).

Tradicionalmente, el medio hídrico se ha considerado como un recurso para el consumo, el aprovechamiento energético, agrícola y el saneamiento (Suárez \& Vidal-Abarca, 2012). Esta visión del río como fuente de recursos y receptor de residuos provocó durante años una percepción negativa del medio fluvial y, esto ha provocado que en muchas ocasiones su función social y medioambiental haya quedado mermada o directamente haya desaparecido. Tras las numerosas investigaciones, que ponen de manifiesto la importancia de los espacios fluviales desde un punto de vista medioambiental (Sánchez et al., 2011; Gómez-Baggethun \& Barton, 2013) y gracias al desarrollo en el campo de la depuración de las aguas residuales urbanas, se produce en España, a partir de los años 80 del siglo pasado, un cambio de paradigma en el que el paisaje fluvial se percibe como un espacio de interés social, cultural y medioambiental (Monclús, 2002; GómezBaggethun \& Barton, 2013; González Rojas, 2017). Además, gracias a la Directiva Marco del Agua (DMA) (Unión Europea, 2000) se ha promovido la restauración fluvial y la mejora del estado ecológico de las masas de agua (González del Tánago et al., 2012). La DMA contribuyó a incrementar significativamente la conciencia medioambiental de muchos gestores de ríos y políticos y propició un escenario de cambio en la concepción de los proyectos fluviales. Para poder alcanzar los requerimientos de la misma, el Ministerio de Medio Ambiente de España desarrolló en 2006 la Estrategia Nacional de Restauración de Ríos (ENRR). Esta iniciativa pretende: (1) fomentar la integración de la gestión de los ecosistemas fluviales en las políticas de uso y gestión del territorio, (2) mejorar la formación de los técnicos, (3) aportar información y experiencias para mejorar las actuaciones y (4) lograr la implicación de los colectivos sociales en la gestión de los sistemas fluviales (González del Tánago et al., 2012; Yagüe Córdova et al., 2010). A pesar de las experiencias realizadas y del avance conseguido en este campo, hoy en día no se alcanzan todos los objetivos que plantea la ENRR, sobre todo en ambientes mediterráneos (González del Tánago et al., 2012).

Esto se complementa con la apuesta de la Comisión Europea por el desarrollo de la infraestructura verde (Comisión Europea, 2014), que favorece la conservación y la restauración funcional entre el medio fluvial y el urbano. Comienza entonces, una nueva etapa de transformación de los ríos urbanos y periurbanos, que se dirige hacia la reconversión del paisaje fluvial ante un nuevo escenario de uso público (Santasusagna \& Tort, 2013; Cheung \& Tang, 2015).

Este cambio de paradigma hace que las actuaciones desarrolladas en los ámbitos fluviales evolucionen desde la creación de espacios públicos netamente urbanos en los que abundan los pavimentos impermeables y zonas ajardinadas con especies alóctonas (Junker \& Buchecker, 2008; 
Cuello, 2009), hacia la renaturalización del medio, a partir de la introducción de pavimentos permeables, estructuras rústicas integradas en el entorno, revegetaciones con especies autóctonas, etc. Es decir, hasta hace pocos años los proyectos de restauración, rehabilitación, mejora y acondicionamiento fluvial se asociaban, sobre todo en grandes ciudades, a obras de reurbanización del espacio ripario (Herrera, 2013), pero, afortunadamente, comienzan a vislumbrarse intervenciones más blandas, más respetuosas con el medio fluvial y a su vez más económicas.

Como casos que hacen referencia al primer escenario, se encuentran el río Segre a su paso por Lleida, el Besós en Barcelona o el Francolí en Tarragona. Respecto al segundo marco, destaca el Arga a su paso por Pamplona o el Zadorra en Vitoria. Estos ríos poseen una mayor naturalidad que los anteriores.

A pesar de estos dos últimos casos citados, en la actualidad todavía son escasos los ejemplos en los que se promueve la integración del río con la ciudad y viceversa (Paredes \& Ballesteros, 2012; González Rojas, 2017). Aun se detectan numerosas carencias y algunas actuaciones son muy cuestionadas ya que emplean soluciones tipo con elevados presupuestos, sin tener en cuenta las especificidades de cada caso y no cumplen con los objetivos que planteaban inicialmente. Además, la mayoría de las obras y actuaciones se presentan como restauración fluvial y no lo son. Esto sirve en ocasiones para evitar procedimientos de evaluación de impacto ambiental o con fines exclusivamente publicitarios (Paredes \& Ballesteros, 2012).

Un caso reciente que ha alcanzado una gran repercusión social y mediática es el de la renaturalización del Manzanares a su paso por Madrid. Este ejemplo muestra la elevada capacidad que tienen los ríos degradados de recuperar sus funciones y servicios mediante la restauración de algunos patrones hidromorfológicos básicos (Magdaleno, 2017). Además, el proyecto ha puesto en marcha un amplio programa de información y participación social activa en cooperación con investigadores del Museo de Ciencias Naturales (Ayuntamiento de Madrid, 2016).

Con respecto a este último, existe un amplio consenso sobre los beneficios que puede aportar la implicación ciudadana a la hora de planificar y gestionar los ríos urbanos (Sánchez et al., 2011; Paredes \& Ballesteros, 2012; García et al., 2017). Sin embargo, esto sigue siendo un reto tanto en España como en otros estados miembros de la UE: no existe tradición participativa en la planificación y gestión de los ríos más allá de la implicación de los usuarios directos del agua o las consultas públicas en una fase avanzada de los proyectos (Sánchez et al., 2011; Heldt et al., 2016). 


\section{Los ríos Arevalillo y Adaja a su paso por Arévalo (Ávila): un espacio de oportunidad}

A continuación, se describe el territorio en el que se enmarca el caso de estudio y se analiza detalladamente la realidad del paisaje fluvial local antes de la ejecución del proyecto.

\subsection{Encuadre territorial}

El municipio de Arévalo está situado en plena llanura castellana, al norte de la provincia de Ávila y limítrofe con las de Valladolid y Segovia (Figura 1). Su ubicación es estratégica puesto que se encuentra cercano a las capitales de provincia circundantes y, aproximadamente, a una hora de Madrid.

Figura 1. Localización del municipio de Arévalo

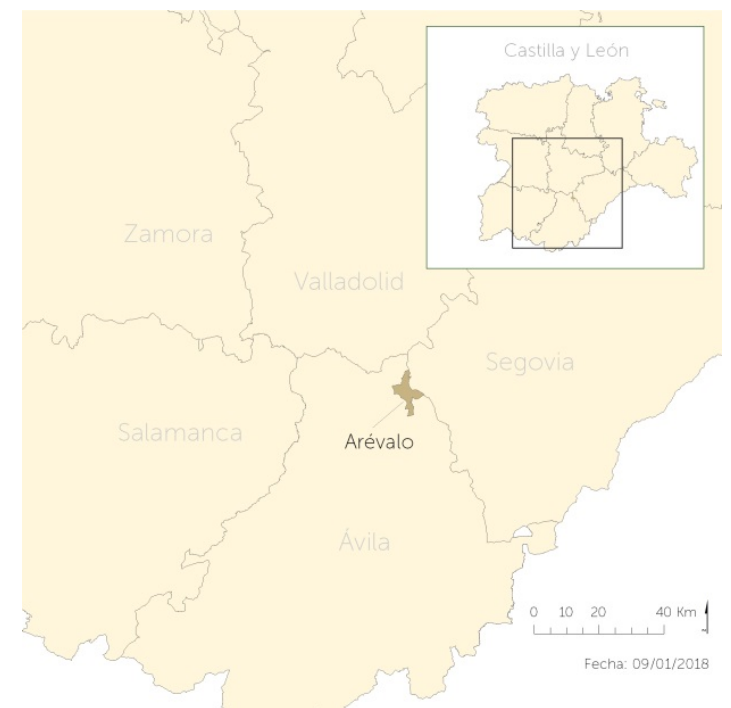

Fuente: elaboración propia a partir de cartografía obtenida

del Servicio de Información Territorial de la Junta de Castilla y León (2018)

El clima es mediterráneo continentalizado, con inviernos largos y fríos y veranos secos, cortos y calurosos. La temperatura media anual es de $12.0^{\circ} \mathrm{C}$ y las precipitaciones anuales rondan los $385 \mathrm{~mm}$ (AEMET, 2018). Existe un fuerte contraste de temperaturas tanto diario como estacional (de hasta $17.9^{\circ} \mathrm{C}$ entre las medias de los meses de enero y julio). Además, las precipitaciones en verano son muy escasas y de carácter torrencial, mientras que el resto del año son más o menos regulares.

La población en 2016 era de 8123 habitantes (INE, 2016). A pesar de ser un municipio rural, alrededor del $56 \%$ de la población activa se dedica al sector servicios. El turismo es una gran fuente de ingresos para Arévalo debido a que existe un importante patrimonio arquitectónico y una 
amplia y reconocida oferta de restaurantes. El número de visitantes anuales va en aumento desde 2014 y actualmente es de 18330 (Oficina de Turismo de Arévalo, 2017).

\subsection{Características del espacio fluvial}

El entorno de Arévalo está constituido por una extensa matriz agrícola salpicada, en algunos puntos, por pinares y choperas de origen antrópico. Apenas se encuentra superficie arbolada a excepción de estas plantaciones y de las formaciones vegetales asociadas a los cursos fluviales que bañan el municipio: el Adaja y el Arevalillo. Los dos ríos, de régimen pluvio-nival y de sustrato fino y arenoso, discurren encajados a ambos lados del casco urbano, confluyendo en la zona más septentrional. De esta forma, se configura un verdadero cinturón verde en torno a Arévalo (Figura 2).

Como río mediterráneo que es, el Adaja presenta un acusado estiaje en verano. A pesar de ello, su caudal al llegar a la localidad de Arévalo es constante, ya que está regulado por el Embalse de las Cogotas, ubicado 35 kilómetros aguas arriba.

Figura 2. Confluencia del Adaja y Arevalillo

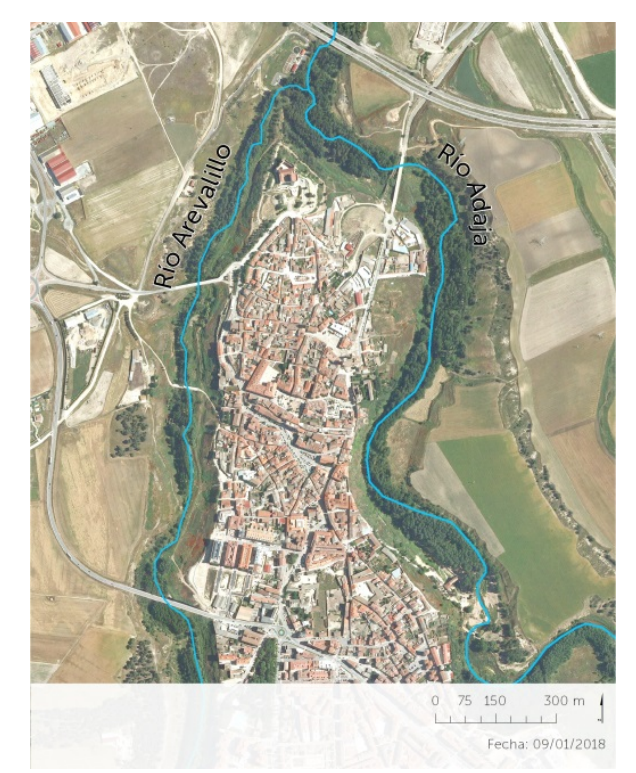

Fuente: elaboración propia a partir de ortofotografía del 2008 obtenida en el Plan Nacional de Ortofotografía Aérea (2008)

Por otra parte, el Arevalillo no está regulado y se trata de un río estacional que, en los últimos años, ha ido adquiriendo un carácter de río transitorio. El principal problema de este curso fluvial es que se encuentra conectado de forma natural con el acuífero de los Arenales y la sobreexplotación de esta masa de agua subterránea provoca que el río sea perdedor durante gran parte del año.

Estos ríos pertenecen a uno de los corredores ecológicos más importantes de la Meseta y único eje verde de la zona norte de la provincia: el corredor del río Adaja. Este conector territorial carece de 
protección legal en la mayor parte de la provincia y su funcionalidad ecológica se encuentra amenazada a su paso por Arévalo, uno de los tramos más fragmentados del corredor.

Acompañando al patrimonio natural, en ambos ríos pueden observarse vestigios históricos y culturales que muestran la estrecha relación entre el ser humano y la naturaleza (Figura 3). Molinos, antiguos puentes medievales y huertas familiares son algunos ejemplos aun presentes en ambos ríos.

Figura 3. Vistas aéreas de Arévalo

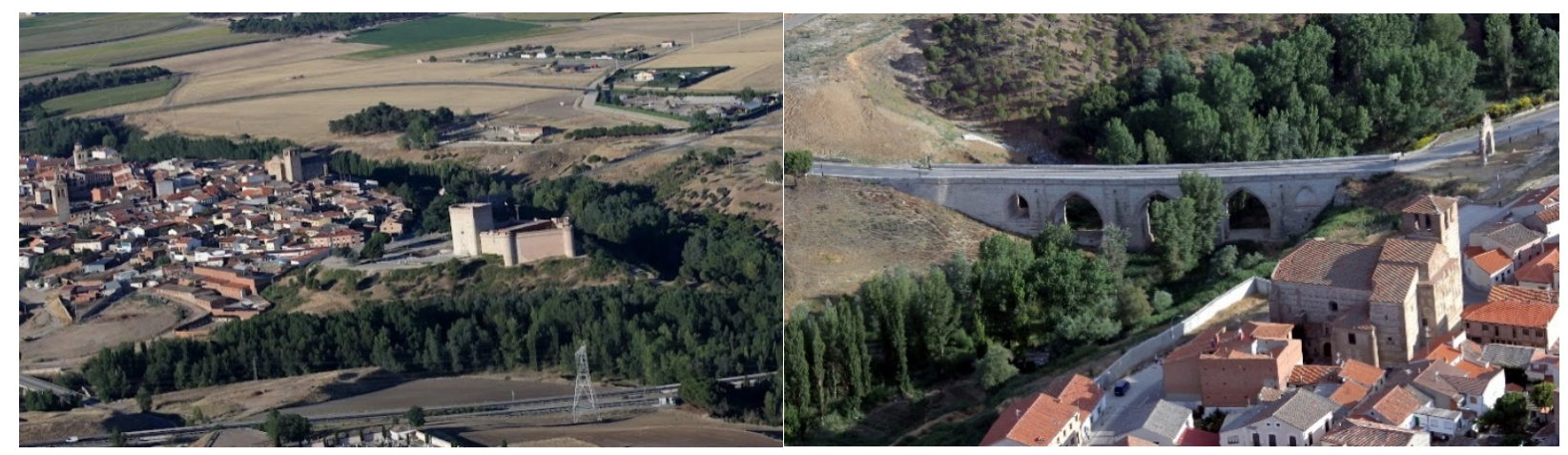

Fuente: Ricardo Melgar (2014)

\subsection{Los problemas del espacio fluvial}

Arévalo es un claro ejemplo de núcleo de población que ha vivido durante años de espaldas a sus ríos. Los usos fluviales tradicionales (industriales, agrícolas, forestales y ganaderos) se abandonaron casi por completo en los años 70-80 del siglo XX y, desde entonces, los ríos -especialmente el Arevalillo- comenzaron a tratarse como espacios secundarios, separados de la realidad urbana.

Este hecho, y la falta de sensibilidad con el medio fluvial, propiciaron que la vegetación herbácea y arbustiva, con alta capacidad de colonización, comenzara a proliferar de forma descontrolada. Además, la desatención de los aprovechamientos madereros conllevó una degradación de las especies leñosas implantadas, que tienen un ciclo de vida corto y que van cayendo progresivamente sobre el cauce fluvial.

La presión de las edificaciones sobre el borde del talud (Figura 4) y la ineficiente canalización de aguas pluviales y residuales procedentes del ámbito urbano, provocaban deslizamientos periódicos en parte de la ladera que, en algunos casos, dieron lugar a desprendimientos de antiguas edificaciones. También, como consecuencia de las continuas deforestaciones efectuadas y los incendios periódicos, se observaba una falta de vegetación arbórea tanto en el valle fluvial como en las laderas (Figura 5). 
Figura 4. Edificaciones sobre el borde del talud del río Adaja

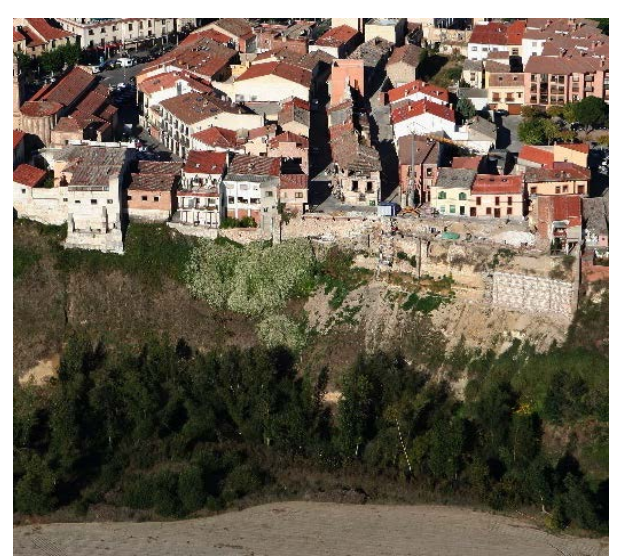

Fuente: Ricardo Melgar (2014)

Asimismo, eran abundantes los escombros y otros residuos sólidos urbanos que se venían vertiendo desde los años 60 del siglo pasado. El cauce fluvial también se encontraba en mal estado, situación que -al menos, en parte- todavía persiste, ya que son varios los aliviaderos que vierten con frecuencia aguas residuales y esto, junto con la ausencia de vegetación arbórea, provoca la proliferación desmesurada de macrófitas con alło poder colonizador (González del Tánago \& García de Jalón, 1995).

Figura 5. Escombros sobre el talud (marzo 2014), vertido de aguas residuales en el río Arevalillo (abril 2014) y restos de un incendio (julio 2014)

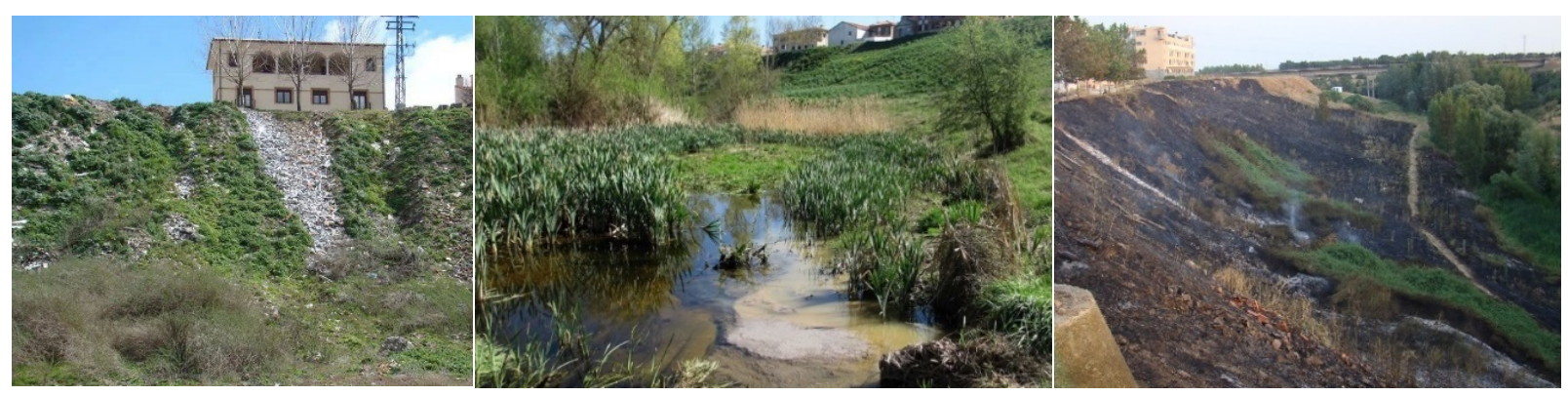

Fuente: Luis José Martín y Francisco Durán (2014)

Todos estos factores hacían que el espacio fluvial fuera un área residual, vacía y desagradable, generando un problema de seguridad, lo que convertía esta zona en un lugar desconocido para gran parte de la población, sobre todo para los jóvenes, abandonado desde el punto de vista social y nada aprovechado, ni por la población local ni por los visitantes.

Ante esta situación, el Ayuntamiento intentó llevar a cabo dos actuaciones en el área fluvial. La primera en la década de los 90 y, la segunda, en el año 2003. En ambos casos, se adecuó un pequeño tramo del río Adaja, próximo al casco histórico de Arévalo, para uso recreativo, pero sin llevar a cabo ningún proceso comunicativo, participativo ni educativo. Se limpiaron las márgenes 
fluviales, se plantaron especies típicas de ribera y se instaló mobiliario urbano (mesas, barbacoas, bancos, alumbrado público, carteles informativos...etc.). Sin embargo, estas actuaciones se descuidaron una vez finalizadas las obras y, hoy en día, las áreas intervenidas se encuentran degradadas, repletas de residuos y son escenario de numerosos actos vandálicos. La zona entonces acondicionada no coincide con el presente ámbito de actuación. Se localiza al sureste del casco antiguo y fue catalogado en el diagnóstico como "área deforestada".

\section{El parque fluvial de Arévalo (Ávila). Rehabilitación paisajística y acondicionamiento para el uso público}

Once años después de la última intervención, nació con un enfoque más amplio y desde el ámbito académico el proyecto "El Parque Fluvial de Arévalo", que pretende recuperar el espacio fluvial local y sus bosques para el uso público, tratando de integrar naturaleza, cultura y paisaje, y procurando mantener, e incluso mejorar, las funciones ambientales del corredor ecológico y, por lo tanto, su identidad natural. Además, debido a la presencia de vestigios históricos en el cinturón, también persigue la creación de un paisaje vivo, no solo en términos ecológicos, sino también culturales.

Por tratarse de un medio parcialmente artificializado, este trabajo no se centra de manera exclusiva en la restauración de los ríos, sino también en su recuperación; es decir, en su mejora y acondicionamiento recreativo. La filosofía que se aplica a lo largo del proyecto es la de trabajar desde la sensibilidad ambiental y social, más que desde la ingeniería tradicional. Sin ser un proyecto de restauración fluvial en sentido estricto (Horacio, 2015), sí se apostó por seguir las directrices dadas al respecto por los especialistas en esta materia. Por ello, se siguió el principio de "no intervención" sobre el cauce (Paredes \& Ballesteros, 2012) ya que se trata de un sistema natural, no regulado y cuyas presiones e impactos son incorregibles e inalcanzables con la ejecución de este proyecto.

Por otro lado, el trabajo se aborda desde una perspectiva multidisciplinar, ya que cualquier actuación que se planifique y ejecute desde una única disciplina en un lugar de características tan peculiares como el referido en esta publicación, donde confluyen agua, riesgos, naturaleza, patrimonio histórico y sociedad, tendría como resultado un enfoque incompleto (Cantó, 2014).

\subsection{Desarrollo del proyecto}

Tal y como se muestra en la Figura 6, la propuesta de actuación derivada de un detallado análisis y diagnóstico territorial, se gestó inicialmente como un trabajo académico realizado en el marco del Máster en Biodiversidad, Paisajes y Gestión Sostenible de la Universidad de Navarra durante el curso académico 2013-2014 (Durán, 2014). Dicho estudio previo, se centró en generar una "imagen objetivo" realista a través del análisis de las presiones e impactos que actúan sobre ambos 
tramos y de los condicionantes ambientales, económicos, sociales, políticos y técnicos que los afectan. Además, investigar en otros tramos fluviales similares y bien conservados ayudó a obtener una "imagen de referencia" y una idea clara de los problemas que padecían los espacios de interés.

Desde el primer momento, además de su función académica, este trabajo tuvo vocación de servir al cambio de la situación en la que se encontraba el espacio fluvial y, por eso, se presentó al Ayuntamiento en 2014 y se dio a conocer a la población del municipio a través de un portal web (https://parquefluvialarevalo.wordpress.com).

Tras ofrecer una estimación presupuestaria cercana a los 200000 euros para la ejecución de la totalidad del proyecto y después de diversas conversaciones, la corporación municipal decidió apoyar su materialización en dos fases: una centrada en el río Arevalillo y otra, en el Adaja. Las características y condiciones de estos ríos difieren entre sí. El Arevalillo, de régimen estacionario $\neg$, presenta una situación más degradada y es ampliamente visible desde el casco urbano, mientras que el Adaja, de régimen permanente, exhibe mejores condiciones, aunque también muestra un acusado abandono social y ambiental.

Tras valorar la situación, el equipo del proyecto decidió concentrar los primeros esfuerzos en la recuperación del espacio fluvial en peor estado de conservación: el del río Arevalillo. En 2015 comenzó la planificación de la primera fase, una etapa en la que se determinaron las actuaciones a ejecutar y el programa teniendo en cuenta, el panorama político, económico, social y ambiental del municipio, asumiendo, al mismo tiempo, cierto grado de incertidumbre respecto al impacto social y acogida del mismo. Justo antes de comenzar la ejecución del proyecto se hizo la primera presentación pública, con cerca de 200 asistentes.

Una vez planificado el trabajo comenzó el proceso de ejecución en el que se agruparon, por un lado, las actuaciones técnicas y, por otro, las de mayor carga social (Figura 6). Primero se materializaron las acciones centradas en la rehabilitación del paisaje y el uso público y al concluir la ejecución técnica, en marzo de 2016, se llevaron a cabo las tareas de educación ambiental, que terminaron a finales de ese año. La participación ciudadana tuvo peso en esta etapa, no obstante, al igual que la comunicación se mantuvo siempre activa.

Por último, este conjunto de acciones fue evaluado a finales de 2016 por la población local a través de una encuesta en la que también se analizaron aspectos de percepción y se preguntó por mejoras e iniciativas que pudieran llevarse a cabo en la segunda fase.

El proceso de comunicación y participación (Figura 6), que permaneció presente en cada una de las etapas, se desarrolló a través de diferentes medios: prensa escrita, radio, vía electrónica (portal web y redes sociales) y entrevistas personales. 
Figura 6. Esquema del desarrollo del proyecto y su cronología

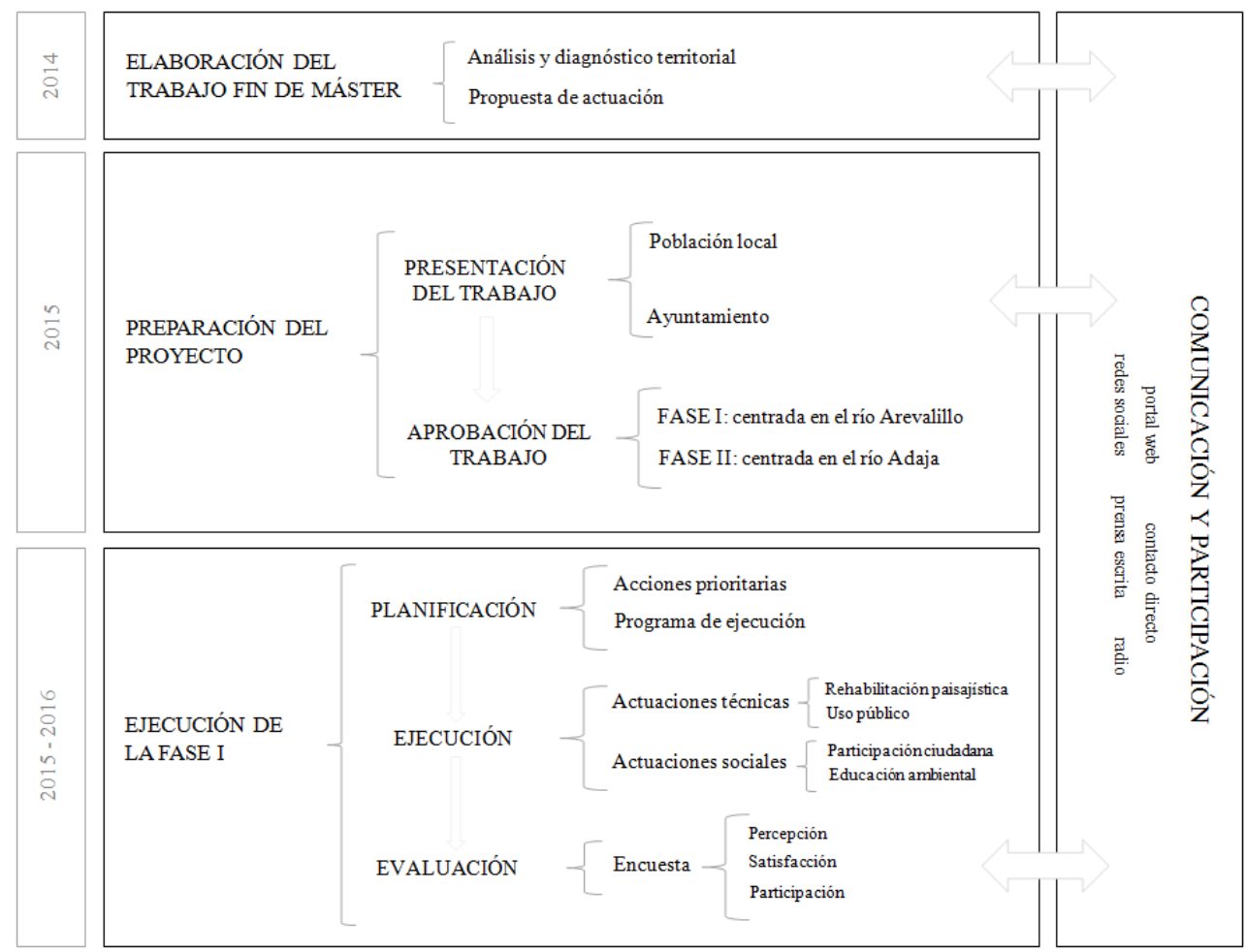

Fuente: elaboración propia

\subsection{Contenido de la propuesta de actuación}

La propuesta se articuló en torno a cuatro estrategias que son: la recuperación de las zonas degradadas, la seguridad, el uso público de las riberas y la comunicación y educación ambiental. Cada estrategia se concretó en una serie de acciones (diez en total) cuyo desarrollo debe servir para impulsar una o varias de ellas (Tabla 1). Estas acciones son (1) tratamiento de las áreas de escombrera y vertederos, (2) forestación de taludes y zonas ribereñas deforestadas, (3) limpieza del entorno ripario, (4) construcción de la senda fluvial, (5) colocación de mobiliario urbano, (6) programa de colaboración, (7) programa de educación, (8) limpieza del cauce fluvial, (9) colocación de pasarelas y puentes de madera y (10) acondicionamiento de áreas recreativas.

Tabla 1. Cuadro resumen acciones-estrategias

\begin{tabular}{|c|c|c|c|c|c|}
\hline & \multirow[b]{2}{*}{ ACCIONES } & \multicolumn{4}{|c|}{ ESTRATEGIAS } \\
\hline & & Recuperación & Seguridad & Uso público & $\begin{array}{c}\text { Comunicación y } \\
\text { educación }\end{array}$ \\
\hline 1 & $\begin{array}{l}\text { Tratamiento de las áreas de } \\
\text { escombrera y vertederos }\end{array}$ & $\bullet$ & $\bullet$ & $\bullet$ & $\bullet$ \\
\hline 2 & $\begin{array}{l}\text { Forestación de taludes y zonas } \\
\text { ribereñas deforestadas }\end{array}$ & $\bullet$ & $\bullet$ & $\bullet$ & $\bullet$ \\
\hline 3 & Limpieza del entorno ripario & $\bullet$ & & $\bullet$ & $\bullet$ \\
\hline 4 & Construcción de la senda fluvial & & & • & \\
\hline
\end{tabular}


Tabla 1. Continuación

\begin{tabular}{|c|c|c|c|c|c|}
\hline & \multirow{2}{*}{ ACCIONES } & \multicolumn{4}{|c|}{ ESTRATEGIAS } \\
\hline & & Recuperación & Seguridad & Uso público & $\begin{array}{c}\text { Comunicación y } \\
\text { educación }\end{array}$ \\
\hline 5 & Colocación de mobiliario & & & • & $\bullet$ \\
\hline 6 & Programa de colaboración & $\bullet$ & & & $\bullet$ \\
\hline 7 & Programa de educación & & & & $\bullet$ \\
\hline 8 & Limpieza del cauce fluvial & $\bullet$ & & $\bullet$ & $\bullet$ \\
\hline 9 & $\begin{array}{l}\text { Colocación de pasarelas y } \\
\text { puentes de madera }\end{array}$ & & & $\bullet$ & \\
\hline 10 & $\begin{array}{l}\text { Acondicionamiento de áreas } \\
\text { recreativas }\end{array}$ & & & $\bullet$ & \\
\hline
\end{tabular}

Fuente: elaboración propia

El tratamiento de las áreas residuales, la limpieza de vegetación no deseada, la forestación y la construcción de una senda de acceso y zonas de descanso han sido las principales acciones materiales en las que se ha focalizado la primera fase del proyecto. Por otro lado, la comunicación, educación y participación social han sido y siguen siendo esenciales para el inicio de una nueva etapa de relación de los ciudadanos con los ríos.

\subsection{Acciones ejecutadas}

Se describen a continuación algunas de las acciones materializadas en la primera fase del proyecto. Las acciones 1 a 5, de carácter más técnico, se condensan en el primer apartado (a) Rehabilitación paisajística y uso público) mientras que la 6 y la 7 , de mayor peso social, se recogen en el segundo (b) Comunicación, participación social y educación ambiental).

a) Rehabilitación paisajística y uso público

En primer lugar, se procedió a la retirada de los desechos sólidos urbanos y escombros presentes tanto en la ladera, como en el fondo de valle (Acción 1). Algunos de los residuos inertes de gran tamaño, procedentes de la construcción y demolición, se colocaron a modo de muro de contención en las zonas de mayor pendiente del talud y, posteriormente, se ocultaron con grandes aportes de tierra vegetal. Fueron necesarios movimientos de tierra de magnitud considerable para ataluzar el terreno. Para luchar contra la erosión y la desestabilización de la ladera se plantaron más de 1500 árboles y arbustos autóctonos (procedentes de viveros locales) de diversas especies (Acción 2), evitando la construcción de escolleras, que hubieran ocasionado un impacto paisajístico negativo. Estas plantas, propias del clima mediterráneo continentalizado, se organizaron en bosquetes aprovechando las vaguadas de la ladera. Cada bosquete o módulo de plantación mixto, está constituido por diversas especies distribuidas irregularmente, pero correctamente espaciadas. 
Destacan en la ladera portes arbóreos como el pino piñonero (Pinus pinea) y la encina (Quercus ilex), combinados con orlas arbustivas de retamas (Retama sp.), jaras (Cistus sp.), aulagas (Genista sp.) y otras aromáticas como la lavanda (Lavandula officinalis) y el tomillo (Thymus vulgaris). En el fondo de valle, desde la orilla del río hacia la zona de ribera, se plantaron sauces (Salix sp.), alisos (Alnus glutinosa), fresnos (Fraxinus angustifolia) y álamos (Populus sp.). Entre los árboles se intercalaron rosales silvestres (Rosa canina) y espinos albares (Crataegus monogyna). Por último, se instaló riego por goteo automatizado en la mayor parte de estos ejemplares a fin de asegurar su supervivencia durante la época estival de los dos primeros años.

Para completar esta actuación paisajística en la ladera se llevó a cabo una hidrosiembra en más de 10 ha de terreno público. Para ello, se utilizaron especies herbáceas de fuertes sistemas radiculares y resistentes a las condiciones ambientales y edáficas de la zona. Entre ellas, destacan Festuca arundinacea, Agropyrum cristatum y Agropyrum desertorum. Además, con el objetivo de mejorar la estética de la ladera durante la estación primaveral se hidrosembró amapola (Papaver rhoeas). En la Figura 8 se aprecia un tramo del espacio ripario, antes y después de las actuaciones.

\section{Figura 8. Valle del Arevalillo en junio de 2014 y en mayo de 2016}

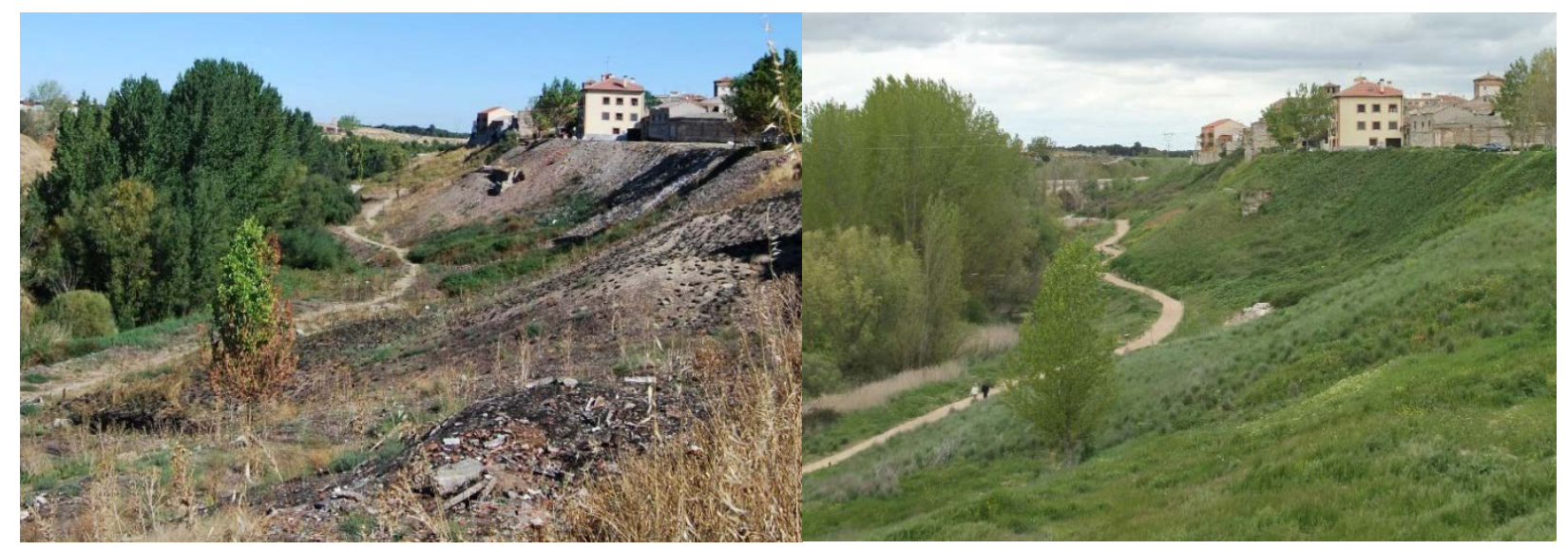

Fuente: Francisco Durán y David Pascual (2014)

Con la finalidad de revalorizar el patrimonio cultural del río se efectuaron desbroces selectivos de la materia vegetal que ponía en peligro y obstaculizaba la visión de antiguos puentes y se ejecutaron actuaciones blandas (Acción 3). Además, utilizando piedras caídas de la antigua muralla, se construyeron tres manantiales que actúan como reservorios de agua natural y donde se ha favorecido la aparición de microhábitats de flora y fauna (Figura 9). 
Figura 9. Puente de los Barros y molino de Valencia, antes y después de las actuaciones
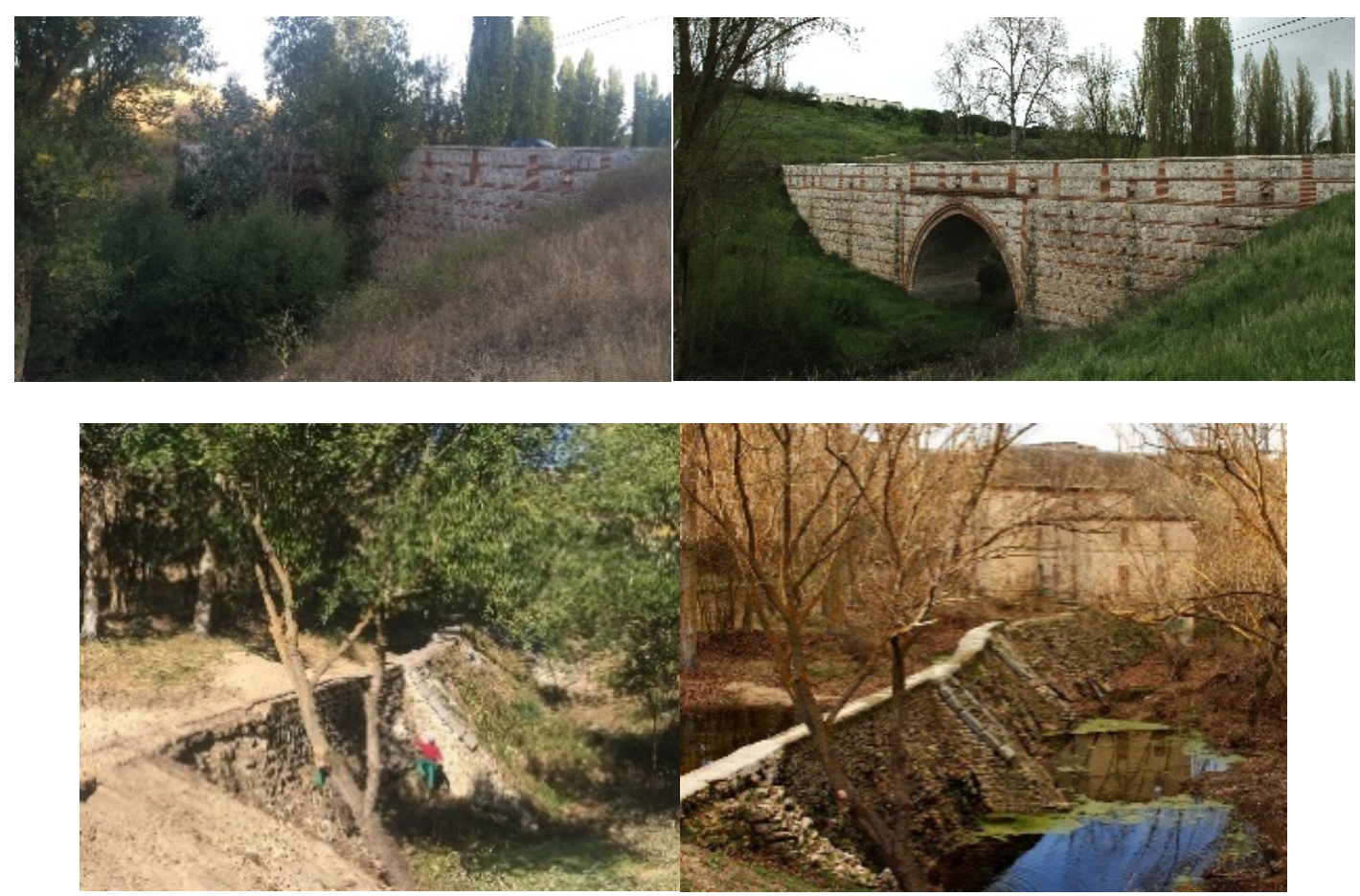

Fuente: Francisco Durán y Julio Pascual (2016)

Por otro lado, se acondicionó un paseo fluvial de 1,6 km de longitud y $2 \mathrm{~m}$ de anchura que comunica el parque fluvial con el casco urbano (Acción 4). El paseo se encuentra a cierta distancia del cauce y está hecho con materiales permeables. En su sinuoso trazado se generan miradores desde los que contemplar los puentes, molinos, presas y otros vestigios históricos presentes en el municipio (Figura 10). Esta actuación, se completó con la colocación de bancos, carteles indicativos y paneles informativos hechos en madera tratada (Acción 5).

Figura 10. Ribera del Arevalillo, antes y después de la actuación de uso público

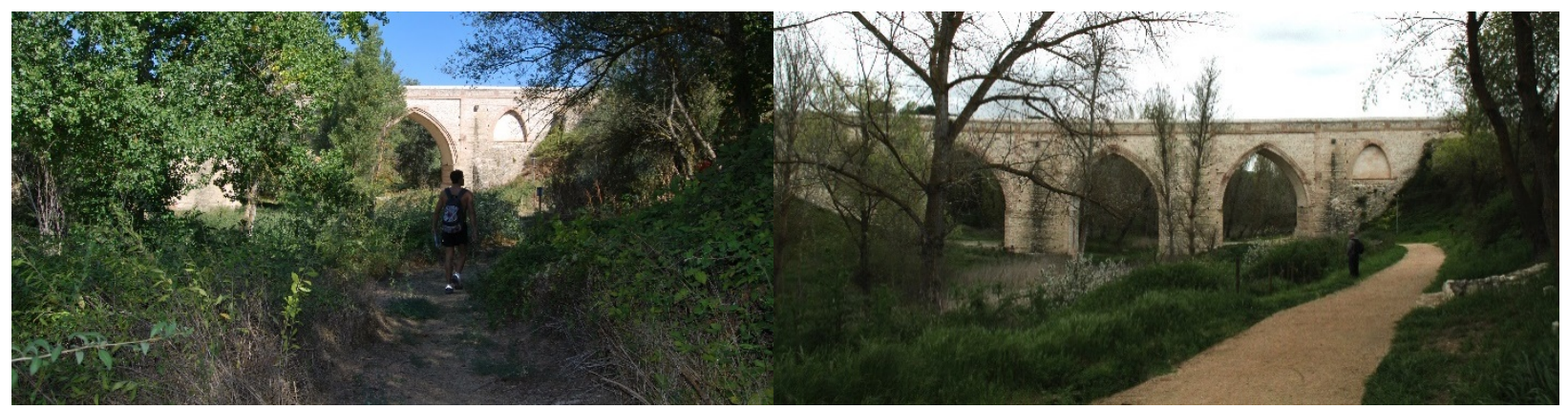

Fuente: Francisco Durán y David Pascual (2015)

Durante la ejecución de esta primera fase se tuvo muy presente la reutilización de materiales; este hecho se concretó, por ejemplo, en la elección del tipo de mobiliario urbano. Así, se emplearon antiguas traviesas de tren y bordillos de granito para escalonar pendientes. Además, se usaron 
como asientos tocones de árboles cortados y grandes sillares de granito procedentes de antiguas edificaciones. Estas muestras de reciclaje presentes en la primera fase, ofrecen a los visitantes un buen ejemplo de sostenibilidad (Figura 11).

\section{Figura 11. Materiales reutilizados para el uso público}
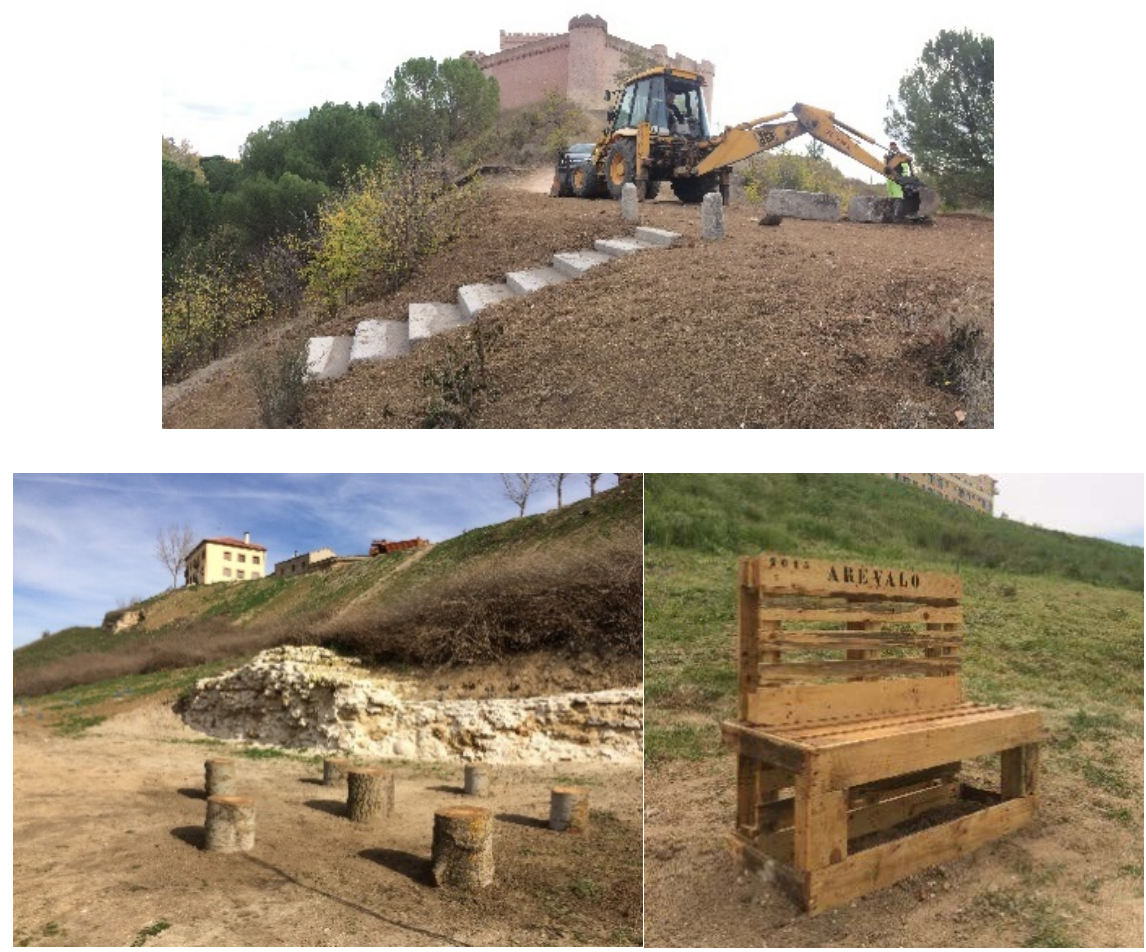

Fuente: Francisco Durán (2016)

\section{b) Comunicación, participación social y educación ambiental}

Los principales objetivos de las acciones de comunicación, participación y educación ambiental fueron, por un lado, dar a conocer el renovado espacio fluvial y así sensibilizar a la población y, por otro, lograr a través de la participación que los vecinos hicieran suyo el parque fluvial, para que así lo respetasen y cuidasen con más empeño.

Antes de comenzar la ejecución de las actuaciones, el trabajo se presentó a la ciudadanía a través del portal web citado anteriormente desde el que los vecinos conocieron las características del proyecto y las acciones a realizar, pudiendo opinar y sugerir propuestas. Además, se celebró con el mismo fin un acto específico de presentación para las asociaciones locales.

Fueron numerosos los ejemplos de colaboración por parte de los ciudadanos de Arévalo durante la ejecución de las distintas actuaciones (Acción 6). A continuación, se comentan algunos de ellos.

Las actuaciones ambientales se acompañaron por obras de un artista local, hechas a partir de metales en desuso, que se contemplan en el cinturón verde y generan un atractivo más del parque fluvial (Figura 12). 


\section{Figura 12. Grupo escultórico "ponte en marcha"}

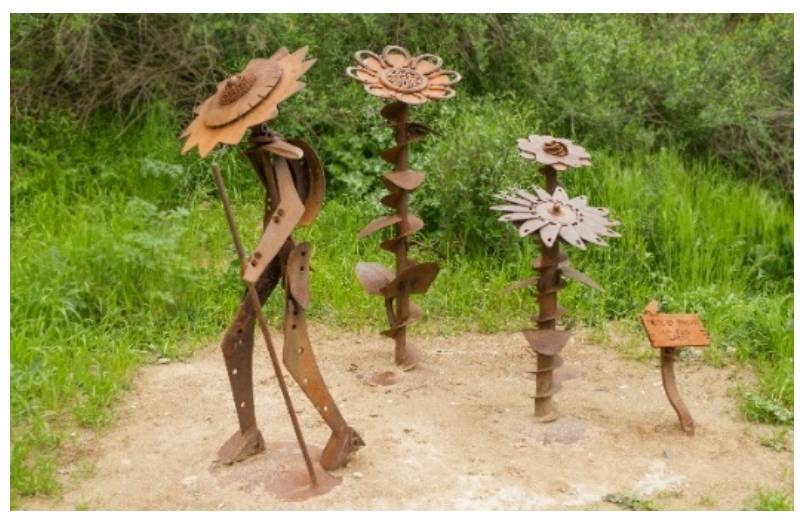

Fuente: David Pascual (2016)

En colaboración con el Centro de Acción Social municipal se organizaron dos talleres dirigidos a minorías étnicas y grupos de exclusión social: uno de jardinería y otro de carpintería. En el primero los alumnos llevaron a cabo gran parte de la plantación y mantenimiento de las especies vegetales y en el segundo (Figura 13), construyeron, a partir de viejas maderas, parte del mobiliario urbano y los carteles informativos. En total, participaron alrededor de 60 personas que, además de adquirir nuevos conocimientos técnicos, aprendieron a trabajar en equipo y pusieron en práctica otras destrezas útiles en el mundo laboral.

Por otro lado, se realizó una jornada de voluntariado ambiental de limpieza y plantación en el río Arevalillo (Figura 13). En este caso, acudieron 90 voluntarios que recogieron más de media tonelada de residuos y plantaron 140 árboles de diferentes especies en la ladera de la margen derecha fluvial.

Figura 13. Alumnos del taller de carpintería y voluntarios en la jornada de plantación

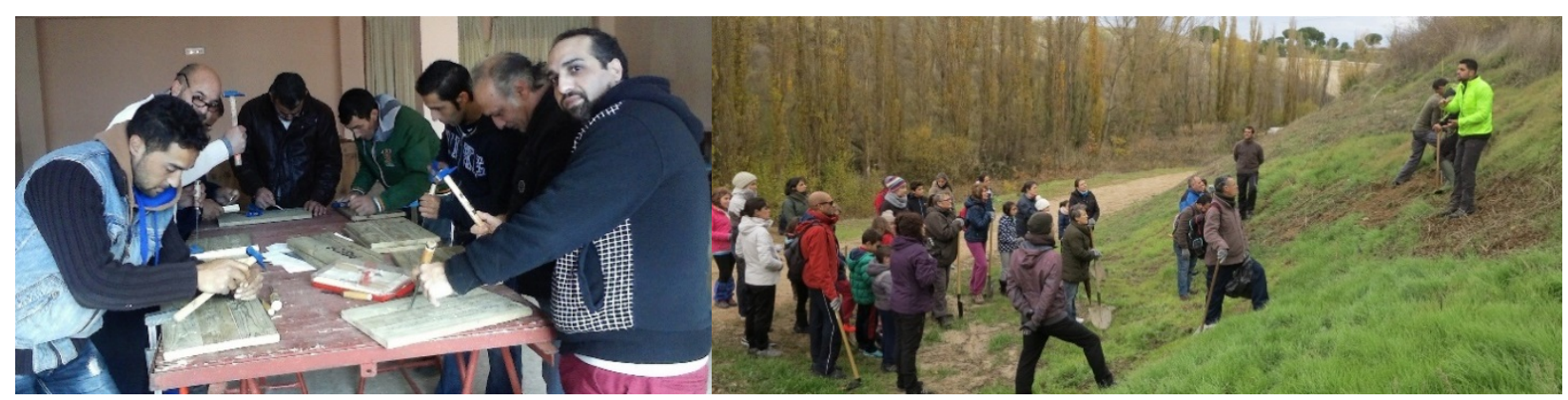

Fuente: Francisco Durán y Ricardo Guerra (2016)

En la Figura 14, que se muestra a continuación, se localizan los problemas identificados durante la fase de realización de la memoria técnica (izquierda) y algunas de las actuaciones desarrolladas hasta la fecha sobre el ámbito de estudio (derecha). 
Figura 14. Zonas degradadas (izquierda)

y actuaciones ejecutadas en la primera fase del proyecto (derecha)

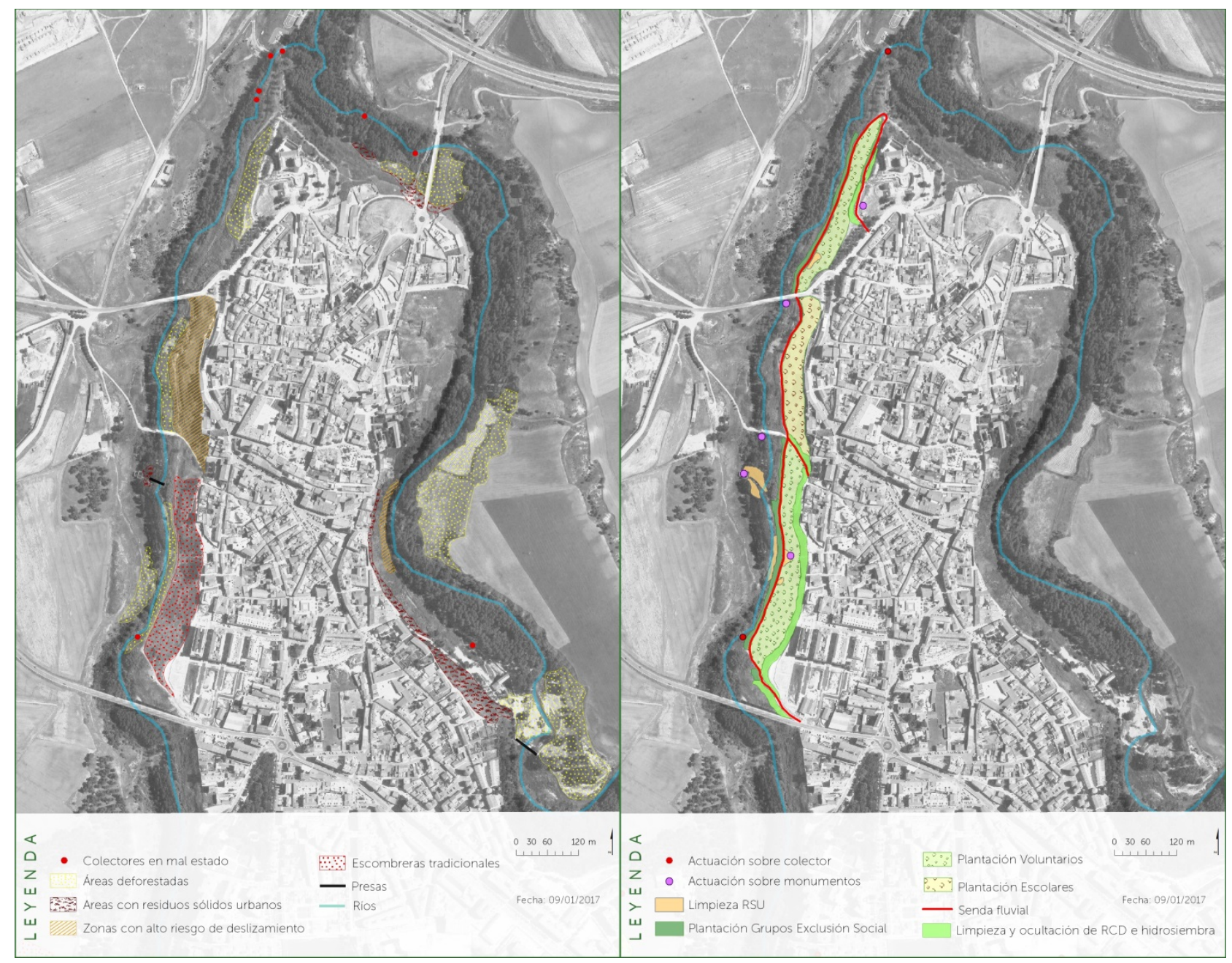

Fuente: elaboración propia a partir de ortofoto obtenida en el Plan Nacional de Ortofotografía Aérea (2018)

Las tareas de concienciación y educación ambiental planteadas en el proyecto fueron diversas (Acción 7). Alrededor de 1300 alumnos de edades comprendidas entre 4 y 18 años y pertenecientes a los 5 centros educativos locales, visitaron el parque fluvial durante el curso. En dichas visitas se comentaron algunas de las intervenciones destacadas y se analizó la importancia ecológica y social del río y sus riberas. Con la colaboración de los profesores de los institutos de educación secundaria se coordinaron talleres botánicos, de análisis de aguas y de suelos, así como de otras materias. También se organizaron, junto con asociaciones vecinales y clubes municipales, otros talleres educativos, rutas ecodeportivas y terapéuticas y eventos deportivos dirigidos al público local, obteniendo altos índices de participación (Figura 15). 
Figura 15. Educación ambiental en el Parque Fluvial y rutas ecodeportivas organizadas por el Ayuntamiento y el equipo de proyecto
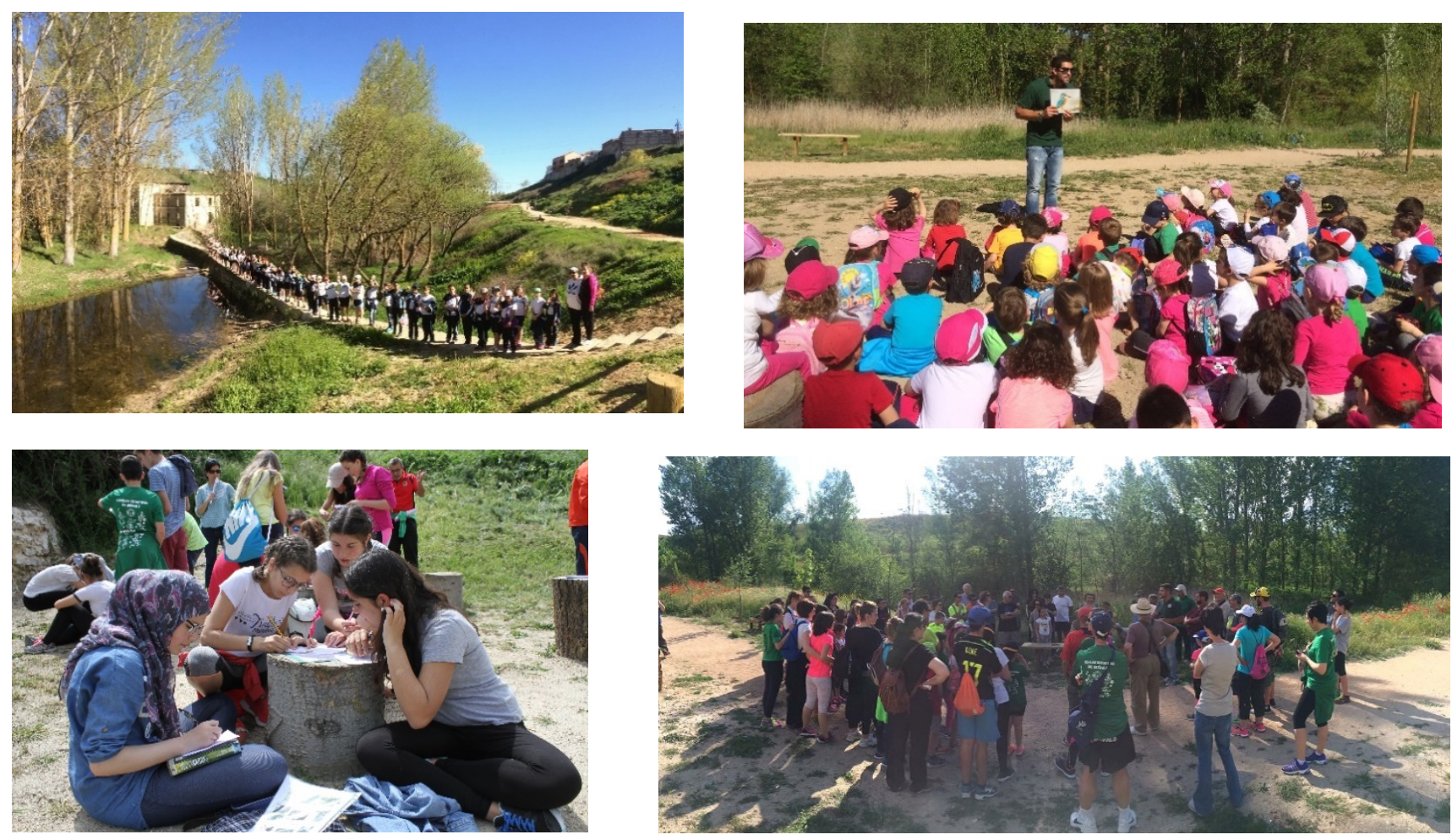

Fuente: Francisco Durán y David Pascual (2016)

Una vez finalizadas las obras de la primera fase, se realizó una encuesta para conocer el grado de satisfacción con lo efectuado hasta la fecha, y para adoptar nuevas propuestas y mejoras de cara a la segunda fase del proyecto. La participación fue muy elevada, recogiéndose un total de 703 encuestados correspondientes a diferentes grupos de edades. La valoración general fue muy positiva. El 84,3\% de los participantes calificaron el proyecto entre "bueno" y "muy bueno". Por otro lado, la encuesta constató que más de la mitad de los habitantes nunca habían acudido al río, situación que cambió tras la ejecución de la primera fase, ya que casi una cuarta parte de los encuestados afirmaron ir semanalmente al paseo fluvial y casi el $40 \%$ dijeron que lo visitan, al menos, una vez al mes.

\section{Discusión}

El caso que se expone no aborda, stricto sensu, un proyecto de restauración fluvial. Al tratarse de un contexło periurbano, es complicado desarrollar un proyecto de restauración propiamente dicho y se ha de asumir cierta artificialidad del medio (González del Tánago \& García de Jalón, 2007). En este caso se ha planteado un trabajo cuya idea no fue la de crear un jardín fluvial destinado al uso público, sino mejorar y acondicionar un área natural con el objetivo de conformar un verdadero "parque fluvial". Según afirman algunos autores, estas actuaciones son poco comunes en la última década (Junker \& Buchecker, 2008; Paredes \& Ballesteros, 2012; González Rojas, 2017). 
El proyecto ha seguido parte de los principios y recomendaciones que numerosos expertos en el campo de la restauración afirman y sugieren en sus trabajos (González del Tánago \& García de Jalón, 2007; Sánchez et al., 2011; Paredes \& Ballesteros, 2012; Herrera, 2013; Ollero, 2015; Horacio, 2015). En primer lugar, se realizó un estudio muy detallado de los tramos de intervención (Trabajo fin de Máster) en el que se evaluaron presiones, impactos y el contexto territorial en general (social, ambiental y económico). A partir de ese estudio se conocieron las condiciones de referencia y se generó una imagen objetivo alcanzable que se persiguió durante la etapa de ejecución. En segundo lugar, se dio un enfoque multidisciplinar, contando con el asesoramiento de políticos, técnicos, propietarios de parcelas ribereñas y otros ciudadanos interesados. En tercer lugar, con objeto de beneficiar al ecosistema fluvial, se respetó una franja ancha de terreno a cada lado del cauce en la cual se limitó la colocación de mobiliario y el paso peatonal. En cuarto lugar, se adoptó el principio de "no intervención" sobre el cauce. En quinto lugar, también se siguieron recomendaciones específicas respecto a la ejecución material (sustrato permeable y anchura de la senda, reutilización de materiales, etc.). En sexto y último lugar, se planteó una intervención basada en la comunicación, participación y la educación ambiental, lo cual ha generado numerosos beneficios de cara a la durabilidad y mejora de lo ejecutado. Gracias a estas acciones sociales, a día de hoy continúan tareas educativas dirigidas por centros escolares y se desarrollan actividades culturales coordinadas por el Ayuntamiento y por asociaciones locales.

Aunque todavía existen dudas sobre los beneficios de la participación ciudadana en proyectos de intervención en el espacio público (Martín-Vide, 2015; Fors et al., 2015), en consonancia con la opinión de algunos autores (Ridder et al., 2005; Turan, et al., 2015; Heldt, et al., 2016), el proceso participativo desarrollado en Arévalo desde el inicio de la planificación del proyecto ha supuesto una vía efectiva para conseguir involucrar a la ciudadanía y encontrar soluciones acertadas. De hecho, la materialización del proyecto en parte fue debida a la presión sobre la corporación municipal que ejerció un grupo de población local.

Como en otros ejemplos (Benages-Albert, 2015a; Benages-Albert et al., 2015b), la participación pública ha contribuido a generar en los arevalenses un sentido de pertenencia hacia el ámbito recuperado. Prueba de ello es que en la actualidad se está utilizando y respetando. Un hecho que, como se comenta en el apartado II.3., no se dio en las intervenciones fluviales anteriores, que se ejecutaron sin un proceso comunicativo, participativo o educativo. De acuerdo con Lorusso et al., (2014), cuanto mayor es el nivel de implicación de los ciudadanos, mayor será su sentido de responsabilidad hacia el espacio.

El éxito social del proyecto se confirmó en la encuesta realizada, ya que un $84 \%$ se considera satisfecho con el trabajo realizado. 
La multidisciplinariedad del equipo también ha aportado resultados favorables. A pesar de que se conocen los efectos positivos que tienen los equipos multidisciplinares y científico-técnicos, son aún escasos los trabajos realizados bajo este escenario (González del Tánago et al., 2012; Ollero, 2015).

Por otro lado, la durabilidad de lo efectuado no solo depende de la sociedad. Una de las grandes carencias del proyecto ha sido la ausencia de planes de mantenimiento y de un adecuado seguimiento técnico, lo que ha ocasionado una falta de gestión directa del espacio por parte de la corporación municipal. Este aspecto se considera vital para el éxito de cualquier actuación de este tipo a medio o largo plazo (González del Tánago \& García de Jalón, 2007) y no siempre está relacionado con la escasez de recursos públicos (Dempsey \& Burton, 2012), sino que puede deberse -como ocurre en este caso- a la falta de previsión de un adecuado sistema de mantenimiento, desde la misma fase de proyecto.

Este aspecto se va a corregir de cara a la segunda fase de la actuación en Arévalo, que contará desde el primer momento con un plan de mantenimiento.

\section{Conclusiones}

A pesar de que aún quedan numerosas actuaciones de carácter técnico y social para recomponer la relación urbano-fluvial, la realización de la primera fase de este proyecto ha supuesto el comienzo de una nueva etapa de reconciliación de los vecinos de Arévalo con sus ríos.

Así mismo, tal y como se corrobora en la encuesta realizada durante el proceso de evaluación, la revalorización del paisaje fluvial ha significado un incremento de la calidad de vida en Arévalo.

Por otro lado, a raíz de la materialización de las acciones recogidas en el trabajo, se ha habilitado un espacio en el que convergen arte, cultura y naturaleza y que constituye un "laboratorio vivo"; un lugar es y podrá seguir siendo testigo de actividades de ocio, educación ambiental, inclusión social y expresión artística.

También es importante remarcar que la implicación de varios agentes locales, liderados y dirigidos por la administración municipal y el equipo multidisciplinar del proyecto, ha sido esencial en el proceso de ejecución, demostrando que los recursos económicos no son tan importantes como la voluntad y el compromiso político y social.

Por último, conviene destacar que el profundo estudio del lugar ha sido esencial para elaborar un buen trabajo técnico fundamentado en la restauración que, junto con grandes dosis de sensibilización y participación, ha logrado la consecución de los objetivos del proyecto.

Agradecimientos: Esta investigación es complementaria al proyecto El Parque Fluvial de Arévalo, cuya ejecución no habría sido posible sin el liderazgo del equipo municipal de Arévalo y sin la 
colaboración de un gran número de vecinos que, tanto desde su perspectiva personal, como profesional, han aportado grandes ideas y han apoyado el trabajo desde el principio.

Declaración responsable: Las/os autoras/es declaran que no existe ningún conflicto de interés en relación a la publicación de este artículo. Francisco Durán ha sido el encargado de realizar la primera redacción del texto, en base al esquema consensuado previamente por las/os tres autoras/es. Miriam Serrano ha aportado conocimientos y referencias bibliográficas para la contextualización del trabajo y la discusión, tarea en la que también han participado Juan José Pons y Francisco Durán. Las fotografías son obra de este último, así como de otros colaboradores ajenos a esta publicación. En la elaboración de la cartografía y de las tablas participaron preferentemente Francisco Durán y Juan José Pons. El diseño de la encuesta que se realizó al finalizar las obras fue coordinado por Miriam Serrano, encargándose Francisco Durán de su distribución y tratamiento estadístico. Las/os tres autoras/es han realizado la revisión de las diferentes versiones por las que ha pasado el artículo y han dado su aprobación al texto final. Una versión inicial de este trabajo se presentó en el encuentro "Caminando hacia la Infraestructura Verde" (20 y 21 de Octubre de 2016), organizado por el Ayuntamiento de Zaragoza en el marco del proyecto LIFE Zaragoza Natural, recibiendo el reconocimiento al mejor póster del evento. 


\section{Bibliografía}

Ayuntamiento de Madrid (2016). Plan de renaturalización del río Manzanares a su paso por la ciudad de Madrid (Report). Retrieved from https://diario.madrid.es/wpcontent/uploads/2016/10/Dossier_Plan_Naturalizacion_Manzanares.pdf

Benages-Albert, M. (2015a). Projectar el Paisatge Suburbà des de l'Experiencia Quotidiana. L'apropiació incremental en la regeneració participada dels corredors fluvials metropolitans (Doctoral dissertation, Universitat Internacional de Catalunya, Spain). Retrieved from https://www.tdx.cat/handle/10803/310951

Benages-Albert, M., Di Masso, A., Porcel, S., Pol, E., \& Vall-Casas, P. (2015b). Revisiting the appropriation of space in metropolitan river corridors. Journal of Environmental Psychology, 42, 115. doi: http://dx.doi.org/10.1016/j.jenvp.2015.01.002

Bolund, P., \& Hunhammar, S. (1999). Ecosystem services in urban areas. Ecological Economics, 29, 203-301. Retrieved from http://dx.doi.org/10.1016/S0921-8009(99)00013-0

Cantó, M. T. (2014). La planificación y gestión de la Infraestructura Verde en la Comunidad Valenciana. Revista Aragonesa de Administración Pública, 43-44, 215-234.

Cheung, D. M., \& Tang, B. (2015). Social order, leisure, or tourist attraction? The changing planning missions for waterfront space in Hong Kong. Habitat International, 47, 231-240. doi: http://dx.doi.org/10.1016/j.habitatint.2015.01.006

Comisión Europea (2011). Green Infrastructure and territorial cohesion. The concept of green infrastructure and its integration into policies using monitoring systems (EEA Technical Report No 18/2011). Luxembourg: Publications Office of the European Union. Retrieved from doi: http://dx.doi.org/10.1016/j.habitatint.2015.01.006

Comisión Europea (2014). Construir una infraestructura verde para Europa. Luxemburg: Oficina de Publicaciones Oficiales de la Unión Europea. Retrieved from http://ec.europa.eu/environment/nature/ecosystems/docs/GI-Brochure-210x210-ESweb.pdf

Cuello, A. (2009). Los tramos fluviales urbanos como ámbito de aprendizaje. Una valoración de su potencial educativo y los obstáculos que plantea su utilización. In M. Junyent, \& L. Cano (Coords.), Investigar para avanzar en educación ambiental (pp. 63-84). Organismo Autónomo Parques Nacionales, Ministerio de Medio Ambiente y Medio Rural y Marino. Retrieved from http://www.mapama.gob.es/es/ceneam/recursos/documentos/Investigar\%20para\%20ava nzar_tcm30-169410.pdf 
Dempsey, N., \& Burton, M. (2012). Defining place-keeping: The long-term management of public spaces. Urban Forestry \& Urban Greening, 11, 11- 20.

Durán, F. (2014). El Parque Fluvial de Arévalo: Propuesta de Intervención Paisajística y Uso Público (Master's Thesis in Biodiversity, Landscapes and Sustainable Management, Universidad de Navarra, Spain). Retrieved from http://dadun.unav.edu/bitstream/10171/45710/1/TFM_Duran.pdf

Fernández, E. M., \& Yáñez, E. (2015). El valor de las áreas periurbanas como espacios multifuncionales en el suroeste de Madrid. Tecnologi@y desarrollo, XIII, separata. Retrieved from https://revistas.uax.es/index.php/tec_des/article/view/614/570

Fors, H., Molin, J., Frøik, M., Melissa, A., \& Konijnendijk Van Den Bosch, C. (2015). User participation in urban green spaces -For the people or the parks? Urban Forestry \& Urban Greening, 14, 722-734. doi: http://dx.doi.org/10.1016/j.ufug.2015.05.007

García, X., Ribas, A., García-Aymerich, J., \& Vall-Casas, P. (2017). Public participation GIS for assessing landscape values and improvement preferences in urban stream corridors. Applied Geography, 87, 184-196. doi: http://dx.doi.org/10.1016/j.apgeog.2017.08.009

Gómez-Baggethun, E., \& Barton, D. N. (2013). Classifying and valuing ecosystem services for urban planning. Ecological Economics, 86, 235-245. doi: http://dx.doi.org/10.1016/j.ecolecon.2012.08.019

González del Tánago, M., \& García de Jalón, D. (1995). Restauración de Ríos y Riberas. Madrid: Fundación Conde del Valle de Salazar.

González del Tánago, M., \& García De Jalón, D. (2007). Restauración de ríos. Guía metodológica para la elaboración de proyectos. Madrid: Ministerio de Medio Ambiente.

González del Tánago, M., García de Jalón, D., \& Román, M. (2012). River Restoration in Spain: Theoretical and Practical Approach in the Context of the European Water Framework Directive. Environmental Management, 50, 123-139. doi: http://dx.doi.org/10.1007/s00267-012-9862-1 González Rojas, D. (2017). Tratamiento de los espacios fluviales urbanos andaluces en la planificación territorial y sectorial. Cuadernos Geográficos 56(2), 72-93. Retrieved from www.redalyc.org/pdf/171/17152020004.pdf

Heldt, S., Budryte, P., Werner, H., Teichgra, B., Schneider, U., \& Deneche, M. (2016). Social pitfalls for river restoration: How public participation uncovers problems with public acceptance. Environmental Earth Sciences, 75, 1053. doi: https://doi.org/10.1007/s12665-016-5787-y

Herrera, T. (2013). Manual metodológico de actuaciones de restauración fluvial y uso público en ámbitos fluviales. Málaga: Diputación de Málaga. 
Horacio, J. (2015). Medicina Fluvial. Un nuevo paradigma en la conservación y restauración de ríos bajo el enfoque de la geomorfología. Lugo: Jolube Consultor Botánico, Jaca \& Fluviatilis River Innovation, S.L.

Junker, B., \& Buchecker, M. (2008). Aesthetic preferences versus ecological objectives in river restorations. Landscape and Urban Planning, 85, 141-154. doi: http://dx.doi.org/10.1016/j.landurbplan.2007.11.002

Lorusso, S., Scioscia, M., Sassano, G., Graziadei, A., Passannante, P., Bellarosa, S., Scaringi, F., \& Murgante, B. (2014). Involving Citizens in Public Space Regeneration: The Experience of "Garden in Motion". In B. Murgante et al. (Eds.), ICCSA, Part II. LNCS, vol. 8580 (pp. 723737). Heidelberg: Springer.

Macdonald, E. (2007). Urban Waterfront promenades and physical activity by older adults: the case of Vancouver. Journal of Architectural and Planning Research, 24(3), 181-198.

Magdaleno, F. (2017). Aesthetic vs. Functional restoration of urban and peri-urban rivers: the Manzanares River in Madrid (Spain). CSE Environmental Design, 1, 48-59. doi: http://dx.doi.org/10.12896/cse201700100103

Martín-Vide, J. P. (2015). Restauración del río Besòs en Barcelona. Historia y lecciones aprendidas. RIBAGUA, 2, 51-60. doi: http://dx.doi.org/10.1016/j.riba.2015.07.001

Mónclus, F. J. (2002). Ríos, ciudades, parques fluviales, corredores verdes. In P. de la Cal, F. Pellicer (Coords.), Ríos y ciudades. Aportación para la recuperación de los ríos y riberas de Zaragoza (pp. 11-31). Zaragoza: Institución Fernando el Católico.

Ollero, A. (2015). Guía metodológica sobre buenas prácticas en restauración fluvial. Manual para gestores. Zaragoza. Retrieved from http://www.revistaesposible.org/phocadownload/guiarestauracion-fluvial.pdf

Paredes, V., \& Ballesteros, F. (Coords.) (2012). Restauración del espacio fluvial. Criterios y experiencias en la cuenca del Duero. Madrid: Ministerio de Agricultura, Alimentación y Medio Ambiente.

Ridder, D., Mostert, H., \& Wolters, H. A. (2005). Aprender juntos para gestionar juntos. La mejora de la participación pública en la gestión del agua» en Proyecto HarmiCOP. Alemania: Universidad de Osnabrück \& Instituto de Investigación de Sistemas Medioambientales. Retrieved from http://www.harmonicop.uni-osnabrueck.de/HCOPmanualespanol.pdf

Sánchez, F. J., García, J., Ballester, A., Molina, J. R., Schmidt, G., López, A., \& Palacios, E. (2011). "Restaurar juntos" - Guía metodológica para proyectos participativos de restauración de ríos. In Actas VIII Congreso Ibérico sobre Gestión y Planificación del Agua "Ríos Ibéricos + 10. Mirando al futuro tras 10 años de DMA (pp. 1-7). February, 16-19, Talavera de la Reina, Spain. 
0003/es/contenidos/informacion/comunicacion_eventos/es_doc/adjuntos/ComunA2/21-

Schmidt.pdf

Santasusagna, A., \& Tort, J. (2013). A propósito de la interfaz ciudad-río. Retos y oportunidades de los espacios fluviales urbanos. In Actas VIII Congreso Ibérico sobre Gestión y Planificación del Agua (pp. 565-575), December 2013, Lisbon, Portugal.

Suárez, M. L., \& Vidal-Abarca, M. R. (2012). Ecosistemas, ríos y riberas: conocer más para gestionar mejor. Revista Ambienta, 98, 134-142.

Turan, S. Ö., Pulatkan, M., Beyazli, D., \& Özen, B. S. (2015). User Evaluation of the Urban Park Design Implementation with Participatory Approach Process. Social and Behavioral Sciences, 216, 306-315.

Unión Europea (2000). Directiva 2000/60/CE del Parlamento Europeo y del Consejo, de 23 de octubre de 2000, por la que se establece un marco comunitario de actuación en el ámbito de la política de aguas. Diario Oficial $n^{\circ} \mathrm{L} 327$ de 22/12/2000. Retrieved from https://www.boe.es/buscar/doc.php?id=DOUE-L-2000-82524

Yagüe Córdova, J., González del Tánago, M., Rodríguez Muñoz, I., Serrano, J., \& Segura Graiño, R. (Coords.). (2010). Bases de la Estrategia Nacional de Restauración de Ríos. Madrid: Ministerio de Medio Ambiente y Medio Rural y Marino. 